\title{
Virtual Reality for Electrical Machine
}

\author{
${ }^{1}$ Nechirvan Salman IBRAHIM, ${ }^{2}$ Erhan AKIN \\ ${ }^{1}$ Computer Engineering, Firat University, Elazig, Turley. \\ ${ }^{2}$ Computer Engineering, Firat University, Elazig, Turley.
}

\begin{abstract}
Virtual Reality (VR) is an innovation that enables a client to collaborate with a computer-mimicked climate, regardless of whether that climate is a recreation of this present reality or a conjured universe. It is the way to find, feel and contact with the past, the present and what is to come. It is the vehicle to make our own reality, our own altered reality. It could go from making a computer game to taking a virtual tour of the universe, from wandering through our own fantasy house to finding a walk on a strange planet. With computer-generated reality, we can meet the most frightening and boring circumstances by playing safe and with a learning point of view.

Mechanical maintenance preparation requires significant investment. Must be legitimate public information personnel before playing out any support on apparent devices and must have an appropriate maintenance and security setting applicable to the specific device support is performed on. Two organizations and scientific institutions face preparing limits when ordering maintenance for replacers or workers.

Virtual Reality situations deal with the issue of access, cost, support, firm plan, and well-being. Virtual reality situations using Game-Engine phases (for this mode, Unity), Computer-Aided-Design display and C\# programming to execute what is needed in a VR reality head where a student is perfectly suited to speak with environmental factors through kidnapping, contact, holding, dropping, tossing and more through existing virtual reality controllers.
\end{abstract}

Keywords: Virtual Reality (VR), Mechanical and Electrical Engineering, 3D Model, AC Motor, Unity.

\section{Introduction}

In recent years, Virtual Reality (VR) became an innovation with the development of created devices that have been used in a number of fruitful exhibits. These days, a wide range of innovations are accessible, for example, Cavern frames, reality theatres, power dividers, holdbacks, individual vivid frames, including Mixed Reality (MR) previews, speech frames, and haptic devices. Furthermore, although specialized frameworks have been improved and expanded, the cost of business forms steadily decreased, consequently developing the ubiquity of the framework among customers, specifically within the modern area.

Simultaneously, Computer Aided tools for engineering purposes (CAX software) presently accessible have been dramatically improved and improved covering essentially all applications in the article life cycle. In any case, the association with the customer within this product is generally done through the CRT/LCD screen and the mouse, which are simply Two Dimensional 2D gadgets. At the same time the mathematical dataset has long been three-dimensional, including the now expanded data, features, and information, the innovation of cooperation has not fundamentally changed since the 1980s [1].

VR is the main theme of the book. In view of the 1990s, the particular innovations have improved essentially and are currently accessible in commercial versions at lower costs, reasonable in any case, for medium and small companies. This topic included three talks and three chosen engagements that offer a decent outline of accessible advancements, as well as expected ways of combining with respect to multimodal interfaces. 
We remained at the doors of another innovative scene. As we watched out at the virtual land attempting to sort out exactly how and what to work there, it was clear there is a great deal to find. In the virtual world, there are no legislative issues or boundaries to what we can insight and what we can work for others to encounter. Similarly as early producers needed to discover how to recount their accounts up on the film screen, we are just barely starting to investigate the sentence structure of Virtual Reality (VR). Indeed, even the actual fundamentals of interfaces: how our kindred cybernauts cooperate with the virtual world, is as yet uncertain. What is the syntax of VR? What feels normal in the virtual world? How would we move around, contact things, feel protests, and control the climate? The quantity of individuals with admittance to the apparatuses to make virtual substance has never been so wide going, and we have more individuals than any other time in recent memory concocting new and energizing increases to our virtual encounters. In the competition to give innovation to oblige new encounters, equipment producers are running as quick as possible to improve VR, more grounded, and quicker. Regardless of how rapidly we race to keep up, the innovation does not quit advancing and evolving. Technology will keep moving. We will never stop learning.

At the point when you arrive at the finish of this paper, it will be dependent upon you to get included and to conceivably begin moulding VR's future. Try not to be reluctant to toss out show and do things that are different to the manners in which we have interfaced with games or re-enactments previously. You are a visionary who could help shape the VR scene of tomorrow. The virtual encounters of things to come will be based upon the encounters of today, so test and make astounding new things! Make sure to have a good time, take care of yourself, and be pleasant to one another.

Virtual conditions or virtual situations are specially crafted three-dimensional virtual universes made out of three-dimensional models, which permit learners to associate and submerge themselves in world that are removed, costly, dangerous, or blocked off. Virtual situations are otherwise called, "artificial reality", virtual worlds and environments. Some Augmented Experience situations, similar to the ones depicted in this paper, presenting a complete immersion in the virtual world through the computer generated simulated headphones from the VR based organizations. [2].

The main programming used during this exploration is known as Unity, and it is the stage of the crossover game engine. It is a product, which permits the advancement of three-dimensional conditions explicitly intended for computer game turn of events; also called a physical science engine. Unity establishes a threedimensional climate with genuine conduct through scripting and full association with the client just as similarity with VR hardware like Oculus Go.

\section{Motivation}

Virtual Reality (VR) is an innovation that gives an intuitive computer-generated climate, generally with a progressively changing situation where one can see and move. VR recreates a client's actual presence in a falsely made world and permits them to communicate with that virtual climate [3]. Most VR applications and arrangements centre on gaming and business ventures, because of these regions giving the biggest gatherings of VR headsets beneficiaries. Nonetheless, the prospects of computer-generated reality do not end with gaming. Dynamic development and interest in the subject of augmented reality have delivered it material in numerous different territories, like the brain science, medication, military, and educating applications. Utilization of data and correspondence advancements has been found to improve understudy perspectives towards learning. Besides, virtual reality assumes a significant part in the showing interaction, giving intriguing and connecting with methods of obtaining data. It can assist educators with disclosing complex issues because of its graphical nature joined with an explorative methodology, actual cooperation and instinctive interfaces.

\section{Technology}

Intended for solace, agreeability, and convenience, Oculus Go is our first independent Virtual Reality item that does not need an outside gadget (PC or compatible mobile phone) to control the experience while in VR. The across the board headset is the most versatile and shareable VR gadget available today, permitting 
individuals to encounter the sorcery of VR, and offer important encounters with individuals they care about, paying little mind to actual distance.

The downplayed outside of Oculus Go conceals imaginative highlights that improve fit and solace, permitting individuals to encounter VR content serenely for longer timeframes. Another framework to station on-board sound nearer to the ears wipes out undesirable link mess and improves the capacity to rapidly get in and out of VR, making it simpler to divide encounters between companions. The aluminium front the gadget assumes two parts, carrying a quality material to the most obvious side of the gadget while scattering heat as a long way from the client as could really be expected. Another direction-followed regulator, streamlined in both structure and capacity, use a portion of the handle calculation, interface points, and trigger ergonomics from Oculus Contact for easy gaming and route.

Expanding on the human components and ergonomic learnings from Oculus Go Fracture, the headset is both instinctive and agreeable. The facial interface was refined to oblige a more extensive scope of face shapes and estimates, and joined with the included glasses spacer, empowers individuals to serenely wear eyeglasses while in VR [4]. Its specifications (Software: Oculus, Hardware: Android, Control: Oculus Go Controller, Motion Detection: 3DOF, Refresh Rate: 72 Hz, Resolution: 1,280 (per eye)).

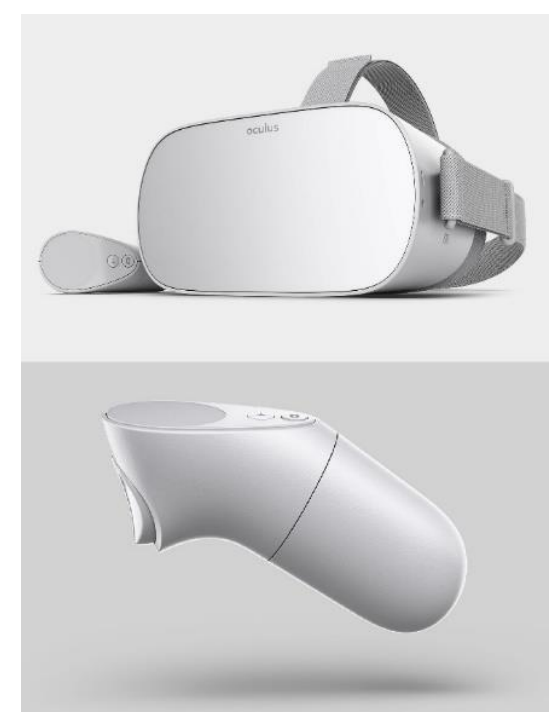

Figure 1: Oculus Go a virtual reality headset developed by Facebook Technologies, Qualcomm, and Xiaomi.

To build up the virtual climate of the model Unity 3D - Game Engine was utilized. This was viewed as the most fitting programming to make VR applications. The programming language utilized for improvement were JavaScript and C\#. To plan and build up all-important virtual articles in the virtual scenes 3Ds Max programming was utilized.

\section{Methodologies}

The venture was directed by Design Thinking (DT) technique, which is an inventive way to deal with create and create thoughts that match the end client's requirements. The iterative cyclic interaction recognizes the cravings of the objective bunch, which they may not know. It depends on instinct, inventiveness, rationale and having a hopeful method to adapt to the difficulties. It has been utilized as a technique for designing items, administrations and encounters with an attention on individuals who will utilize them, gotten from five organized stages sympathize, characterize, and ideate, model and test [5]. 


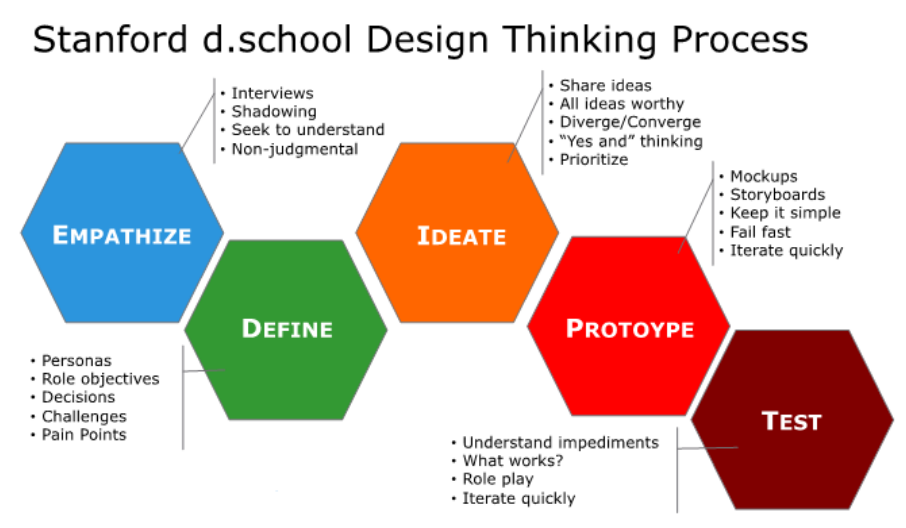

Figure 2: Design Thinking (DT) process.

\section{Planning Specifications}

The initial step of the advancement cycle begins with the plan details. This climate includes a real application in an ideal area, and these prospecting centres on mechanical support. Just as it is practiced, the mechanical cycle or machine that will give rise to the hypothetical reaction must be examined and realized. When the fashioner has a decent comprehension of the cycle, details, instruments on whichever part of the interaction the instructional exercise will be made for, machines and actual parts required are set up, and the initial step is finished. I examined the situation in these centres and a paper about disassembly and re-get together of AC (Permanent Magnetic Synchronous Motor PMSM), (See Figure 3).

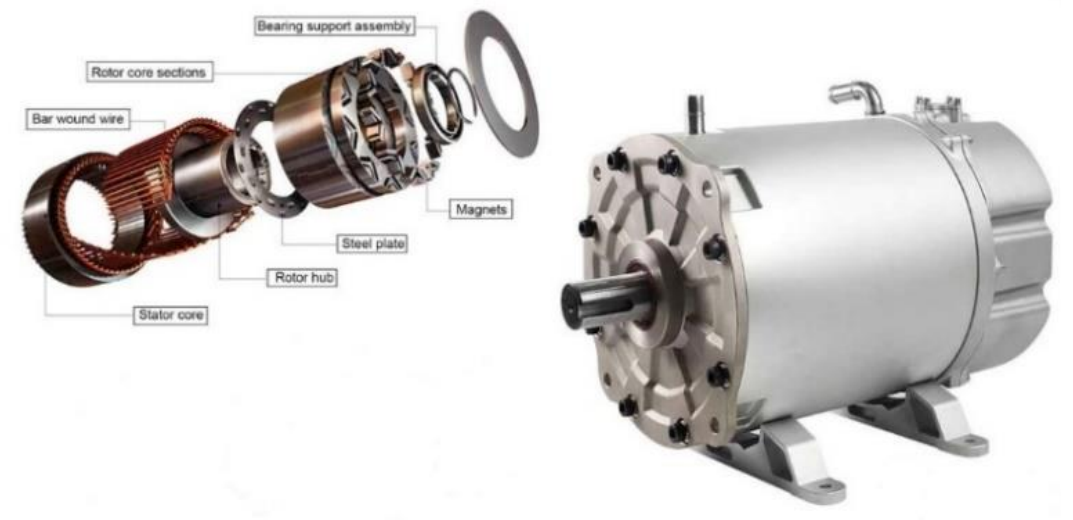

Figure 3: Motor Components.

Alternating Current (AC) is an electric motor that is driven by an alternating flow.AC motors are commonly used in mechanical settings for various applications including but not limited to absorption, blowers and transmissions, which is why AC electric motors are essential for various tasks regardless of work. In the absence of motor failures, it can mean extravagant personal time because it is essential to ensure plants run easily and adequately. Focus on its maintenance. A special cycle with the help of this motor is to replace the rusty bearing. The motor selected for this setup includes some parts that must be taken out to use the bearings [6].

Table 1: Motor Parts to be disassemble.

\begin{tabular}{|l|c|}
\hline Parts to be removed & Quantity \\
\hline Screw & 1 \\
\hline Stator-Cover & 2 \\
\hline Fan-Cover & 1 \\
\hline Fan & 1 \\
\hline Bearing & 1 \\
\hline Magnets & 1 \\
\hline
\end{tabular}




\begin{tabular}{|l|l|}
\hline Rotor-Core-Section & 1 \\
\hline Steel-Plate & 1 \\
\hline Rotor-Hub & 1 \\
\hline Rotor & 1 \\
\hline Bar-Wound-Wire & 1 \\
\hline Stator-Core & 1 \\
\hline
\end{tabular}

The parts recorded in Table 1 must be eliminated in the correct descending order. In addition, a realistic drive requires the bolts and nuts to be dislodged before the means shown in Table 1; however, this hypothetical position is lacking in small equipment. The cycle of identifying the ideal application that would be hypothetical is extensive. There are many varied possibilities for compiling virtualization applications. For this paper, the application has chosen the need to satisfy some of the basic requirements that need to be considered for selection.

In the next part of the step, a plan is discussed that focuses on selecting the devices required for a given reaction. For this situation, dismantling the motor to access the old parts will be done physically without the use of devices. It was a specific mission, and the ultimate goal of this situation was to replace the old parts. The customer has an option to play with this project effectively, in fact, as in the virtual climate.

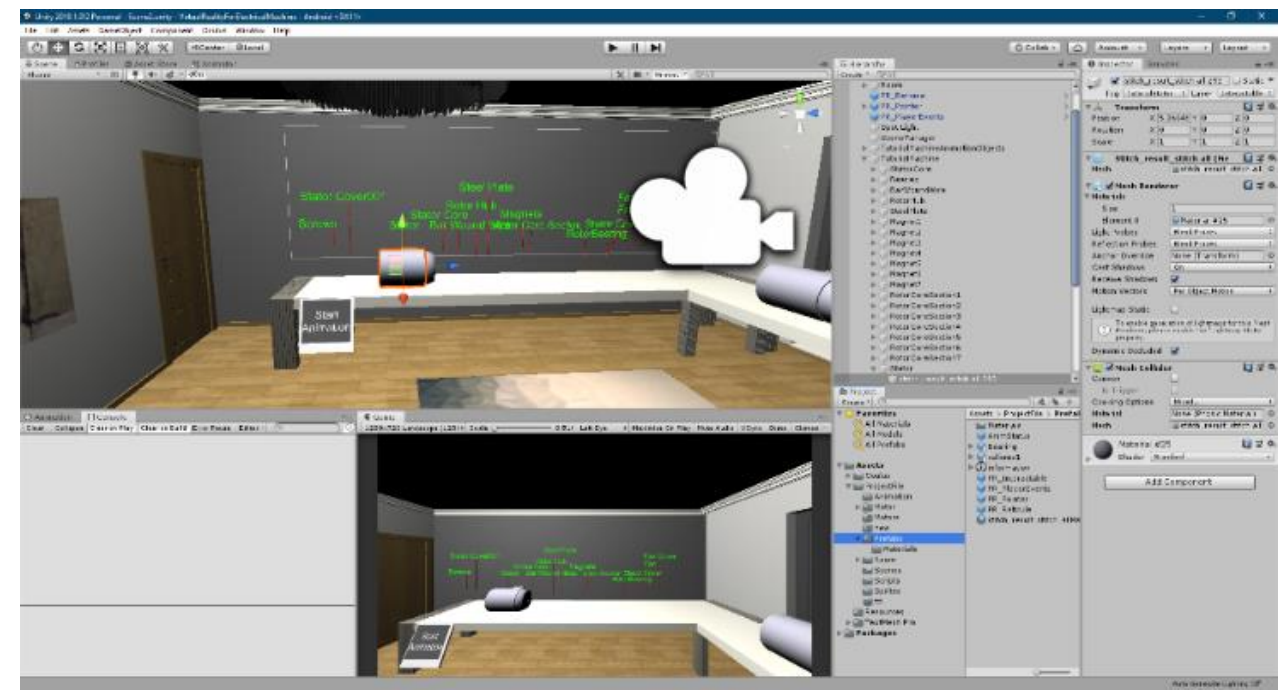

Figure 4: Motor in the Virtual Scenario (Unity).

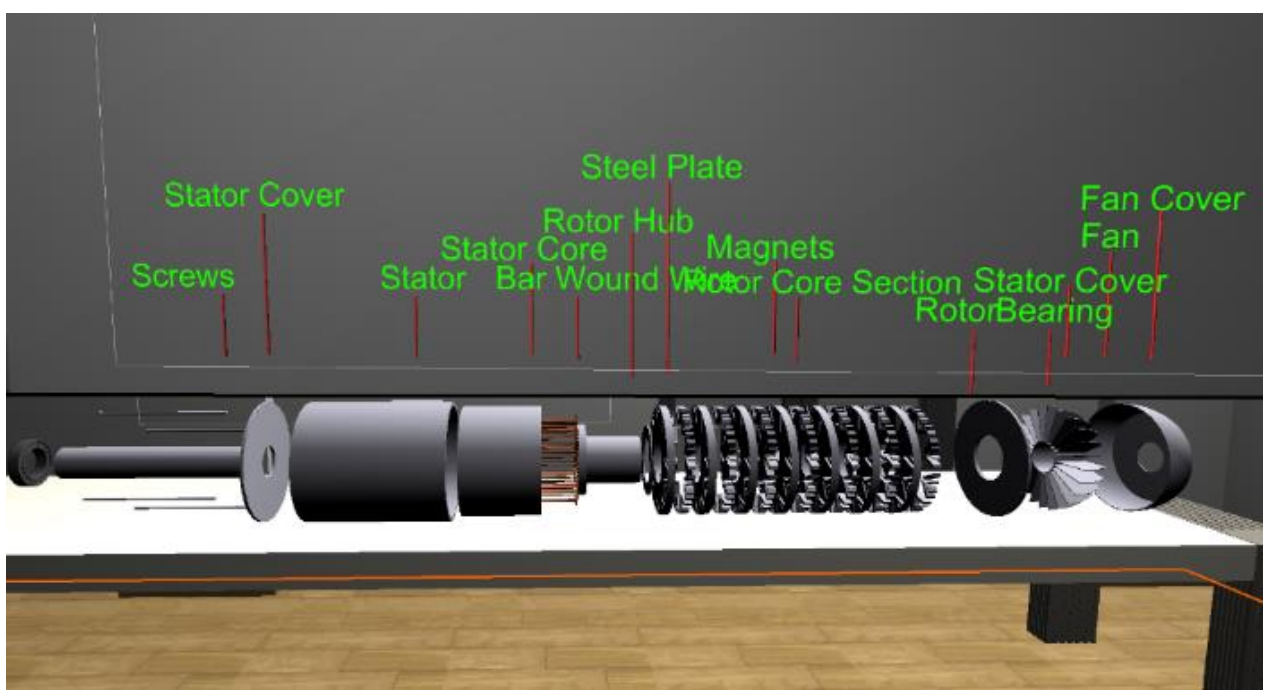

Figure 5: Motor components in the Virtual Scenario (Unity). 
The customer needs to be familiar with the device and use it effectively in the virtual climate to have the option to effectively finish the method. This will finally show the customer the right device for the decided application. More devices will be inserted in mode for visual purposes, and some of them will run.

\section{Three-Dimensional (3-D) Models}

The second part of the interaction begins with the simple recording of the essential parts of improving the situations. Parts can be given either by existing 3-D CAD models or can be made by 3-D modelling software, for example CATIA, Rhino3D, Maya, 3-Ds Max, SolidWorks, AutoCAD, SketchUp, Blender, ZBrush, etc. (see Table 2). (See Figure 6). Summarize and look at the big discrepancies of these 3-D modelling tools. Most of the commonly used modelling tools are skilled and used in their modern applications.

Not only are these devices used in a CAD (Computer Aided Design) plan, but they also provide some highlevel light, for example, (Computer Aided Engineering) for performance testing [7], additive manufacturing and three-dimensional printing [8].

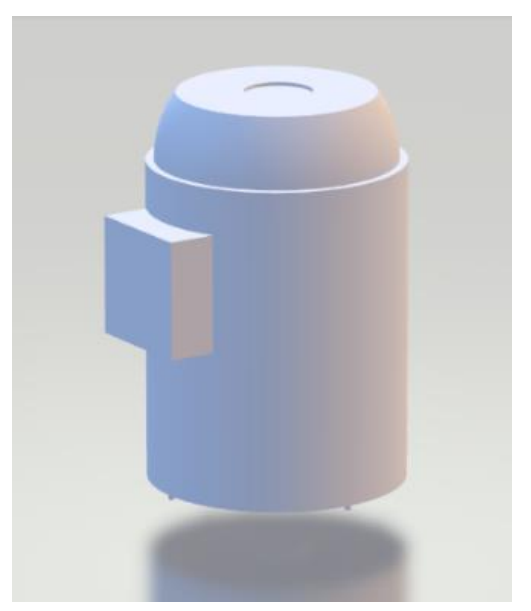

Figure 6: 3-D Model motor.

Table 2: Comparison of major modelling software.

\begin{tabular}{|l|c|c|c|}
\hline $\begin{array}{l}\text { Modelling } \\
\text { Application }\end{array}$ & Type & Convenience & File Extension \\
\hline CATIA & CAD & Pro. & .x3d,.vrml97,.vrml,.stl,.ply,.svg,.lwo,.x,.obj,.fbx, \\
\hline Rhino3D & CAID & Pro. & .skp,.ply,.off,.obj,.rws,.lwo,.kmz,.igs,.gts,.gdf,.fbx, \\
\hline Maya & CAID & Pro. & .stl,.obj,.fbx,.dxf, \\
\hline 3Ds Max & CAID & Pro. & .nx,.jt,.ipt,.iges,.flt,.dwf,.dxf,.catpart,.catproduct, \\
\hline SolidWorks & CAD & Pro. & .vrml,.stl,.stp,.pdf,.obj,.ifc,.idf,.dxf,.dwg,.amf, \\
\hline AutoCAD & CAD & Pro. & .pdf,.dxf,.dwg, \\
\hline SketchUp & CAD & Begin. & .stl,.kmz,.ifc,.def,.dem,.dae,.3ds,.dxf,.dwg, \\
\hline Blender & CAID & Int. & .vrml,.stp,.sldasm,.sldprt,.skp,.sat,.rvt,.prt,.prj,.obj, \\
\hline ZBrush & CAID & Pro. & .x3d,.vrml,.stl,.obj,.ma,.goz,.dxf, \\
\hline
\end{tabular}

\section{Mesh Disentanglement}

Solid works make weighing three-dimensional (3D) models that are made of thousands of different triangles, lines, and shapes (See Figure 7). This really makes a detailed logbook of great importance. While making the interpretation of this document to increase coherence for a virtual environment like FBX, we have experienced quite a bit of trouble. For this interpretation, when a three-dimensional (3D) model is obtained for the perfect part or rendering, the lattice is rearranged by making use of unexpected software in comparison to that of three-dimensional modelling (3D), for this mode (3D StudioMax; product by Autodesk) For modelling, animation and rendering). A (3DS Max 2017) A grid is a variety of vertices, 
edges and faces that depict a state of a three-dimensional (3D) item. Vertex being a single point each threedimensional (3D) model consists of networks. The more confused the poetic, the more the point by a point the better the item will glance at the last situation virtual reality, but more complex cross-sections for the equivalent heavier than a large document. Matches also take into account three-dimensional (3D) elements to give surfaces and shadows, and to give the finished situation a more realistic look. Three-dimensional (3D) models with a ton of cross-sections will make inconveniences when bringing a few without a moment's delay in to the drive and unit game maker. Each component must be changed separately and then imported. When the article has been sent to from 3D StudioMax to the unit, the cross sections cannot be modified. This means that a cycle between a game engine and 3D StudioMax must be completed several times until an ideal state is found. "Sweet spot", where the material will be not enough stout a large glance at the apparent climate yet light enough for the unit to supervise it, it must be found. The entanglements in the game engine maker occur because of unity must present every element that the individual wearing virtual reality is taking a gander at, just as check the actual behaviour of these materials gradually. Dependence on a piece of material that needs to be transferred or have different tones and surfaces must be separated mesh items. In the event that a piece of a three-dimensional (3D) model would not be important to applying an ideal educational practice the item could either be dispensed with or rearranged. The game engine has a specific file type that it accepts, so 3D Studio Max software additionally allows changing the file type from models with endings like .SLDPRT or .SLDASM to .FBX, which is the type that the unit supports. (See Figure 7) shows an illustration of an element with two different network properties. The most distant item to a franchise has a large network, which adds a lot of detail [9].

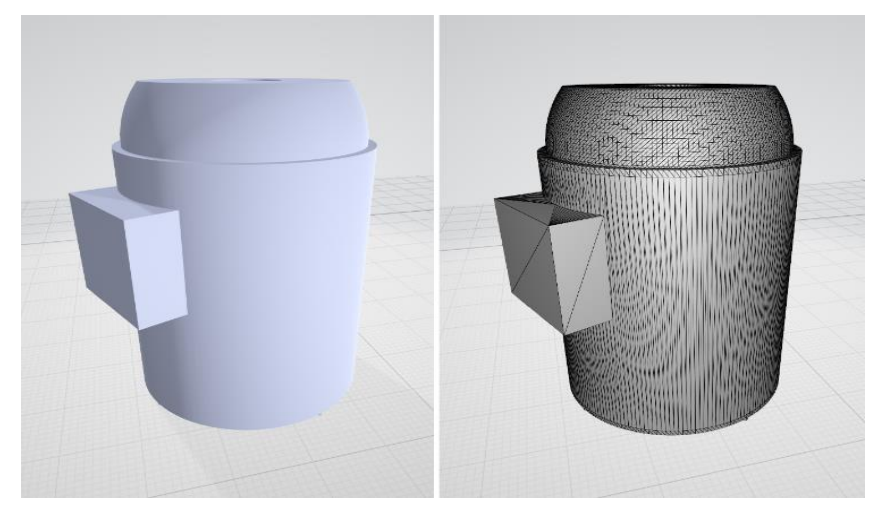

Figure 7: 3-D Mesh Comparison Close-up.

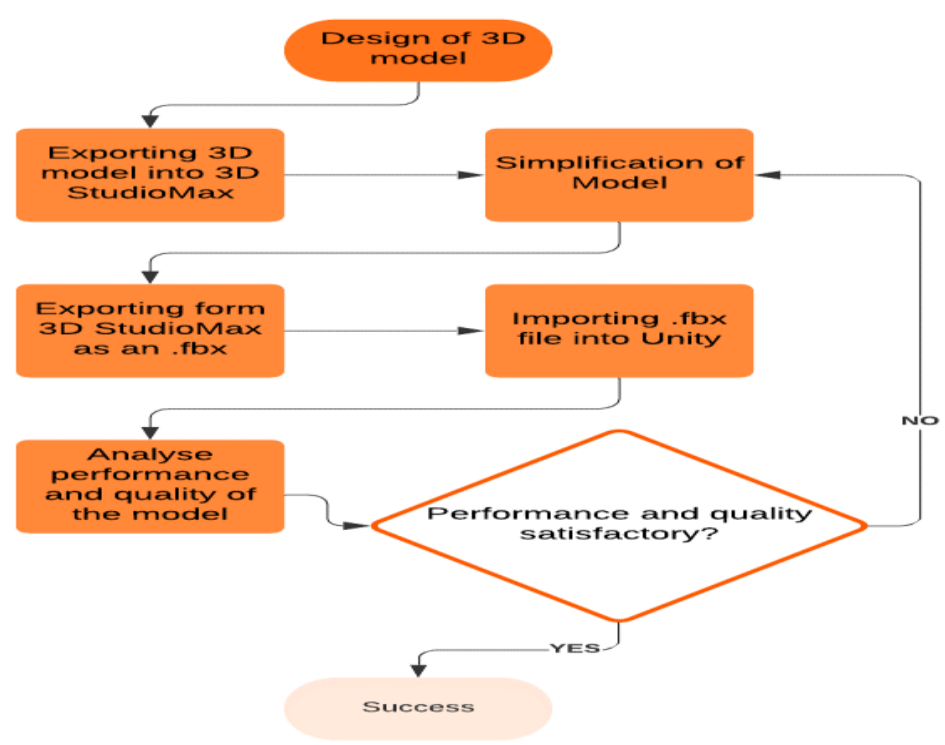

Figure 8: A Virtual Reality program development flowchart. 


\section{Building a Main Menu with Unity GUI}

We look at how to build a main menu Scene. As well as building the user interface (UI), we look at three methods for interacting with UI in virtual reality (VR) and scripting the behaviours to correctly load another Scene when the Start Game button is activated.

UI, for VR, is a new art that we are only just beginning to understand. Old methods of flat screens with buttons feel unnatural in a virtual world, forced in there through necessity rather than to enhance an experience. Some of the best VR interfaces are the ones that act as a part of the universe they inhabit. If an interface has rules that tie into the virtual universe and make sense, it can be much more natural to use.

\section{Tutorial Machine Table}

Click on the table (Tutorial Machine), when you click the start button (Start Animation) (See Figure 9a), the motor parts will be separated (See Figure 9b). Display the motor part name when clicking on the part (See Figure 9c, d).

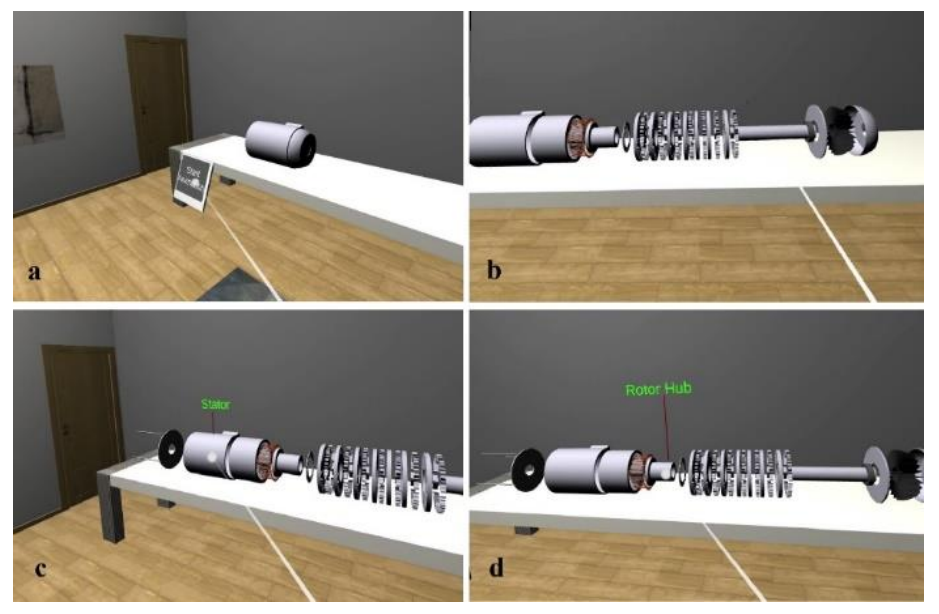

Figure 9: The main menu to start project (Tutorial Machine) (a) Start button (b) Separate part. (c) Select stator. (d) Select rotor hub.

\section{Assembly Machine Table}

Click on the table (Assembly Machine), when you click on the cell of the table (6- Bearing) (See Figure 10a), the motor bearing part will be separated, and the motor part name will be displayed.
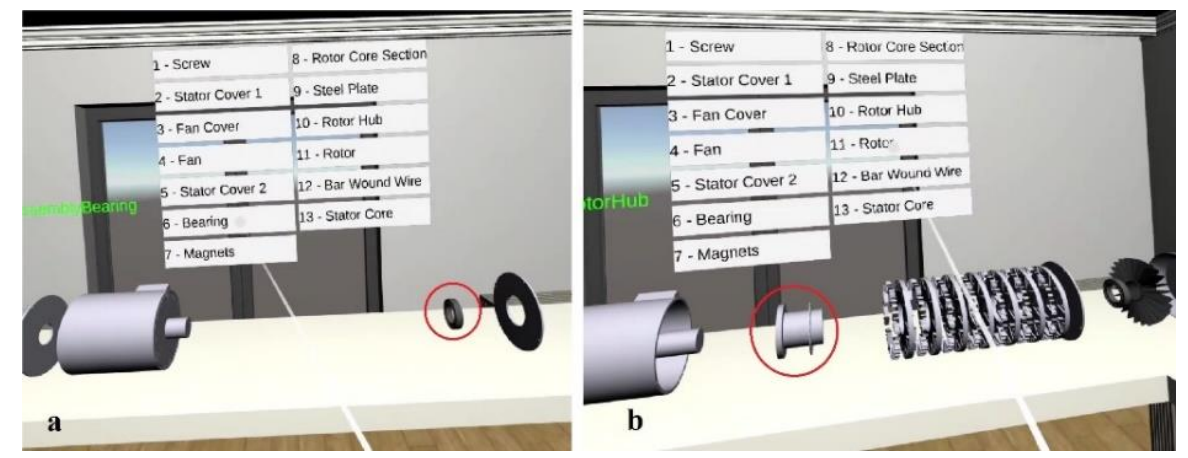

Figure 10: The part separated (Assembly Machine) (a). (6- Bearing) (b) (10- Rotor Hub).

\section{Maintenance Machine Table}

The last step, click on the table (Maintenance Machine) you can allow the user to assemble a part when the part is assembled into the appropriate system according to the (First In Last Out FILO) algorithm. 


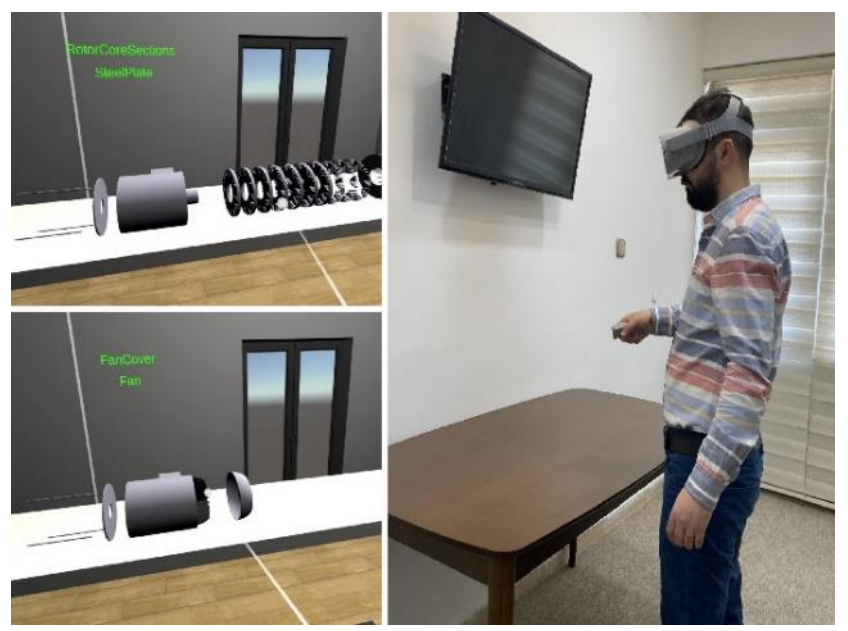

Figure 11: User in the testing process the assembled part (Maintenance Machine).

\section{"THAT'S NOT THE NEXT PIECE!"}

The last advance in the structure of the Augmented Simulation for Electrical Machine is transforming the game into an instructional exercise is finished by scripting just as by carrying out show remark. In programming, it permits the client to acknowledge when mistakes happened by executing the remark show "THAT'S NOT THE NEXT PIECE!" When some unacceptable part is held or not permitting the client to gather a section when the part is not collected in the right request, every development order is enacted when the past advance is finished accurately right.

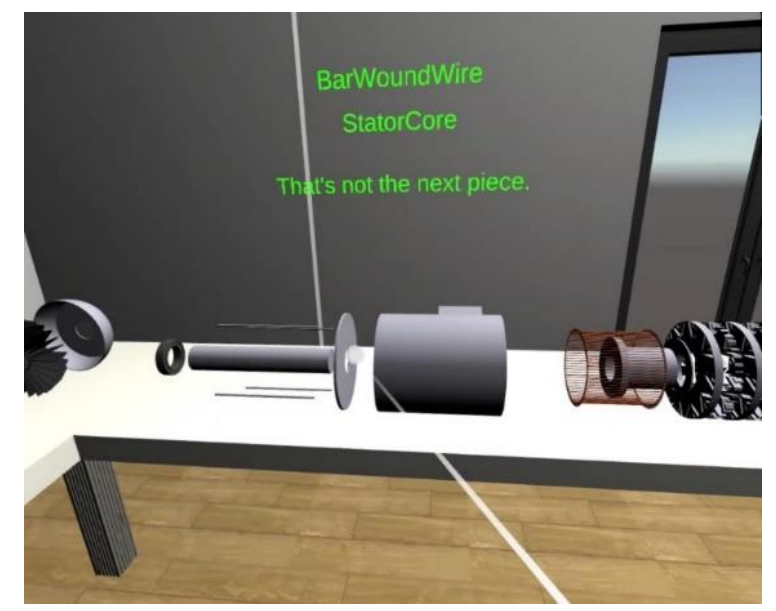

Figure 12: Display comment when wrongly assembled part.

The instructional exercise project likewise incorporates the scripting of the motor dismantling activity instructional exercise (See Figure 12) which permits the client to see how the motor is dismantled. At that point, the client can genuinely choose the parts, which show their names (See Figure 12). This shows the client the parts, their area inside the motor and their names.

\section{Programming}

When every one of the articles have colliders and actual qualities, their conduct identified with the client can be customized. The vast majority of the actual conduct that identifies with cooperation with the client just as developments must be modified utilizing scripting. As Unity states on their site:

"Programming is quite basic defining games. In fact, even the slightest requirements of the game difficult contents, to respond to user interference and harmony occasions in constant, interaction occurs when they have to. Then, the contents can be used to create graphic effects, and control the actual behaviour of the objects". 
Unity takes into account two Java and C \# programming dialects. C \# was used to implement the project (Virtual Reality for Electrical machine). Software engineers can pick among C\# and Java relying upon experience or inclination since both have a similar functionality [10]. A portion of the capacities that were modified utilizing C\# in Unity for the Electrical Machine situation includes:

- The robotized generation of the gathering and dismantling of a motor, that fills in as an instructional exercise.

- The association between the clients that permits snatching parts in the climate like pieces of the motor for dismantle and collect.

- The connection of the client and the apparatuses, the association among devices and parts.

- The framework that permits the motor to be just amass and dismantle in the right request.

Through the Unity creator window (See Figure 13), we can observe which motor the customer needs to disassemble. In the right side of the window, we can see a portion of the devices that this particular GameObjects has. This complex content allows the customer to extract each piece separately to the motor and collect just as it is disassembled. Content window consists of a few factors. Unity makes these factors into content and really shows them to see behaviour and examine programming as it occurs continuously.

The total scrip in $\mathrm{C \#}$ is given underneath for instance:

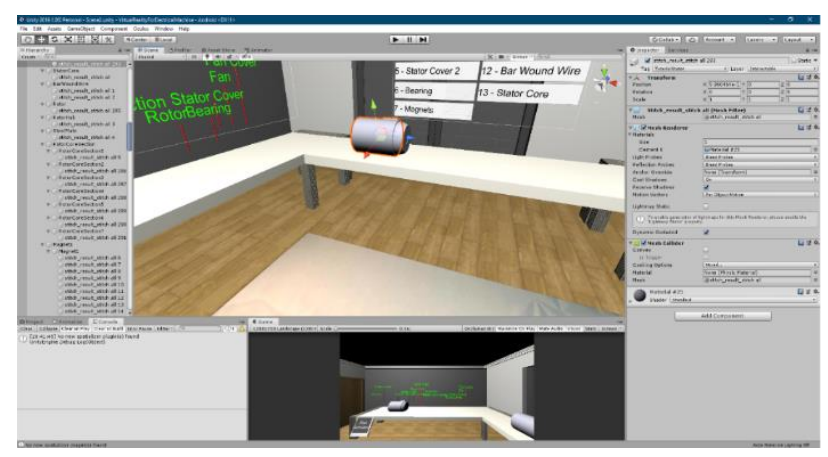

Figure 13: The complete scripts.

\section{Unity GUI}

Essentially, anything that you can make with the regular Unity GUI system is up for grabs in VR. The system we will be using here will just hook into Unity's existing systems to do the actual UI work, leaving SteamVR to deal with detecting when the controller is trying to interact by using scripts provided as part of the SteamVR library [11].

\section{Discussion and conclusion}

The aim of this paper was to make the VR for Electrical Machine that was reasonable and able to prepare the individual to play a particular strategy in a safer manner without any requirements that the now-daily regular preparation techniques provide. The overall goals of this paper are where they met. Initially, the mechanical support scale was recognized. This cycle has answered all necessities, in order to produce a fruitful preparation device. Interactivity was seen completely, devices expected to play out by mechanical maintenance, all things considered, so he admitted. The authenticity of the situation was accomplished and even outperformed assumptions utilizing surfaces, mood articles, lightning and then some. The climate ends up being more secure without the utilization of any actual hardware, which likewise permits the situation to be accessible consistently. The conduct of the Virtual Situation was modified utilizing C\#; the conduct gives both usefulness and authenticity to the virtual preparing situation. The production of an instructional exercise to prepare an individual the correct request of a mechanical support technique was accomplished utilizing a 3-venture measure. The first step was the educational exercise movement that showed the customer an activity in the course, with the various steps for deconstruction and the names of the parts. Subsequent advances, which allow the customer to perform the said action using physical connectivity 
through the designated organizers Oculus Go that have allowed the customer to acquire, select and play the devices just like chips without effort.

This case has proven to be an exceptional tool for education as for modern preparation, as an additional device. All through the paper may be different combinations of substitutes, teachers, and talking employees got the opportunity to use the virtual mode. Entrance obtained from a wide variety of encounters on various levels was positive. This situation has appeared on three occasions, high school enrolment has changed one suitable school choice where it has always been the main charm for substitutes only as teachers. Virtual reality has long been used for gaming purposes, given that games are many dollars. This has caused many organizations to put a large number of dollars into this innovation. The application of gaming innovation, as we have seen with this paper, can be used merely as a useful purpose-setting mechanism, allowing computer games to provide a more important cause in life. This study showed that the preparation situation is reasonable to take place. A situation like this takes into consideration the individual to learn in a more efficient manner. This virtual situation demonstrates that we can prepare more individuals at a lower cost and in a safer way while taking advantage of the advancement of simulating existing additions. This situation did not put restrictions on access to materiel, restrictions on spending and social welfare issues.

\section{References}

[1] D. Talaba and A. Amditis, Product engineering: tools and methods based on virtual reality. Springer Science \& Business Media, 2008.

[2] A. Alaraj et al., "Virtual reality training in neurosurgery: Review of current status and future applications," Surgical Neurology International, vol. 2, no. 1, 2011, DOI: 10.4103/2152-7806.80117.

[3] D. A. Bowman and R. P. McMahan, "Virtual Reality: How Much Immersion Is Enough?" Computer, vol. 40, no. 7, pp. 36-43, 2007, DOI: 10.1109/MC.2007.257.

[4] W. Greenwald, “Oculus Go Review,” PCMag, Jun. 23, 2020. .

[5] Brown and Tim, "How Design Thinking Transforms Organizations and Inspires Innovation," 2009. Accessed: Mar. 06, 2021. [Online]. Available: https://www.goodreads.com/book/show/6671664change-by-design.

[6] J. W. Krakauer and R. Shadmehr, "Consolidation of motor memory," Trends in Neurosciences, vol. 29, no. 1, Jan. 2006, DOI: 10.1016/j.tins.2005.10.003.

[7] Y. M. Tang, A. Zhou, and K.-C. Hui, "Comparison of FEM and BEM for interactive object simulation," Computer-Aided Design, vol. 38, pp. 874-886, Aug. 2006, DOI: 10.1016/j.cad.2006.04.014.

[8] S. Stadler and M. Hirz, "A knowledge-based framework for integration of computer aided styling and computer aided engineering," Computer-Aided Design and Applications, vol. 13, no. 4, pp. 558569, Jul. 2016, DOI: 10.1080/16864360.2015.1131552.

[9] R. F. Tobler and S. Maierhofer, "A Mesh Data Structure for Rendering and Subdivision,” 2006.

[10] J. W. Murray, "C\# Game Programming Cookbook for Unity 3D,” 2014.

[11] Jeff W. Murray, "Building Virtual Reality with Unity and SteamVR," 2020. 